\title{
Comparison of Kerr and dilaton black hole shadows
}

\author{
Jan Röder, ${ }^{a, b, *}$ Alejandro Cruz-Osorio, ${ }^{a}$ Christian M. Fromm, ${ }^{a, b}$ Yosuke Mizuno,,${ }^{a, c}$ \\ Ziri Younsi $^{d, e}$ and Luciano Rezzolla ${ }^{a, f, g}$ \\ ${ }^{a}$ Institut für Theoretische Physik, \\ Max-von-Laue-Straße 1, D-60438 Frankfurt am Main, Germany \\ ${ }^{b}$ Max-Planck-Institut für Radioastronomie, Auf dem Hügel 69, D-53121 Bonn, Germany \\ ${ }^{c}$ Tsung-Dao Lee Institute and School of Physics and Astronomy, \\ Shanghai Jiao Tong University, Shanghai, 200240, China \\ ${ }^{d}$ Mullard Space Science Laboratory, \\ University College London, Holmbury St. Mary, Dorking, Surrey, RH5 6NT, UK \\ ${ }^{e}$ UKRI Stephen Hawking Fellow \\ ${ }^{f}$ Frankfurt Institute for Advanced Studies, \\ Ruth-Moufang-Straße 1, 60438 Frankfurt am Main, Germany \\ ${ }^{g}$ School of Mathematics \\ Trinity College, Dublin 2, Ireland \\ E-mail: jroeder@itp.uni-frankfurt.de, osorio@itp.uni-frankfurt.de, \\ cfromm@itp.uni-frankfurt.de, mizuno@itp.uni-frankfurt.de, \\ z.younsi@ucl.ac.uk, rezzolla@itp.uni-frankfurt.de
}

The vicinity of supermassive black holes (SMBHs) has been in the focus of scientific research for decades. Open questions revolve around the types of compact massive objects in the centers of galaxies, plasma dynamics around them and emission processes at play. The goal of this study is to assess whether it is possible to distinguish between two spacetimes in observations of such objects and their environment by means of synthetic imaging. To this end, general relativistic radiative transfer (GRRT) calculations are carried out on general relativistic magnetohydrodynamics (GRMHD) simulations of a Kerr- and of a non-rotating dilaton black hole. We parametrize the proton-to-electron temperature ratio and analyze the source morphology. From the GRMHD simulation and GRRT images, we conclude that differences are hard to detect in a real-world observation scenario, especially for jet-dominated images. The differences can be explained by the absence of rotation in the dilaton system and the matching of the black holes at their innermost stable circular orbit.

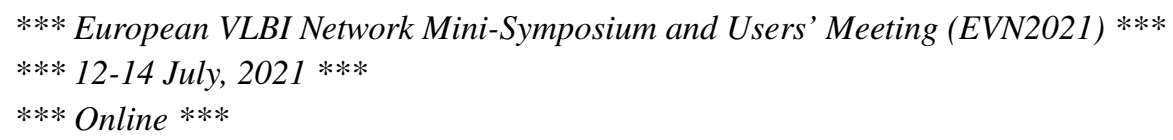

${ }^{*}$ Speaker 


\section{Introduction}

Ever since the discovery of quasars in the early 1960s, studies of active galactic nuclei (AGN) have been a prominent field in astronomy [1]. Soon, black holes were generally accepted as the root of the observed extreme-energy output of some galaxies. Over the decades, evidence for the existence of supermassive black holes was first found indirectly through movement of stars in the galactic center [2,3], and the existence of stellar-mass BHs was confirmed by the first detection of gravitational waves [4]. Most recently, through the developments in millimeter-wavelength very long baseline interferometry [5], the Event Horizon Telescope Collaboration obtained a direct image of the shadow of the SMBH at the heart of M 87 [6].

Ever since it was first published [7], Einstein's theory of general relativity has been put to the test countless times [for a detailed review, see e.g. 8]. The goal of this work is to assess whether a Kerr black hole can be distinguished from a dilaton black hole by means of magnet-hydrodynamic modeling in full general relativity and subsequent radiative post-processing.

\section{Methods}

\subsection{General-Relativistic Magneto-hydrodynamics (GRMHD)}

Following the pioneering work of Mizuno et al. 2018 [9], we choose as representative systems a Kerr black hole with dimensionless spin $a_{\star}=0.6$ and a non-rotating dilaton black hole with dilaton parameter $b=0.504$ in spherically symmetric polar coordinates. The latter black hole is described by the Einstein-Maxwell-dilaton-axion (EMDA) gravity [10], with the axion field set to zero. Setting $b=0$ would recover the Schwarzschild metric. The two black holes are matched at the innermost stable circular orbit (ISCO).

The GRMHD equations consisting of local conservation of mass, energy and momentum along with Faraday's law read [11, 12]:

$$
\begin{array}{r}
\nabla_{\mu}\left(\rho u^{\mu}\right)=0, \quad \nabla_{\mu} T^{\mu \nu}=0, \quad \nabla_{\mu}{ }^{*} F^{\mu \nu}=0 \\
T^{\mu \nu}=\rho h_{\mathrm{tot}} u^{\mu} u^{v}+p_{\mathrm{tot}} g^{\mu \nu}-b^{\mu} b^{v}, \\
{ }^{*} F^{\mu \nu}=b^{\mu} u^{v}-b^{v} u^{\mu}
\end{array}
$$

In Eq. $1, \rho$ is the rest mass density and $u^{\mu}$ is the fluid four-velocity. Equations 2 and 3 show the energy-momentum tensor $T^{\mu \nu}$ and the dual of the Faraday tensor ${ }^{*} F^{\mu v}$, with total pressure $p_{\text {tot }}=p+b^{2} / 2$ and specific enthalpy $h_{\text {tot }}=h+b^{2} / \rho$. Lastly, $b^{2}=b^{\mu} b_{\mu}$ and $b^{\mu}$ describe the magnetic field strength in the fluid frame and magnetic field four-vector. The spacetime metric $g^{\mu \nu}$ either takes the form of the Kerr metric, or the aforementioned EMDA dilaton metric.

Both GRMHD simulations are initialized with a static hydrostatic equilibrium torus with a constant angular momentum distribution $[11,13]$. The torus is governed by an ideal gas equation of state with adiabatic index $\Gamma=4 / 3$, while on the outside pressure and density floor values are applied [9]. The gas pressure in the torus is perturbed by $1 \%$ in order to trigger the magneto-rotational instability, which starts and drives the accretion of gas onto the black hole.

We add a poloidal magnetic field onto the constructed torus, with the vector potential in the form $A_{\phi} \propto \max \left(\rho / \rho_{\max }-0.2,0\right)$. This magnetic field configuration will produce a Standard and Normal 


\begin{tabular}{llcl}
\hline \hline \multicolumn{4}{c}{ Plasma parameters } \\
adiab. index $\Gamma$ & density floor $\rho_{\mathrm{fl}}$ & pressure floor $p_{\text {gas, fl }}$ \\
\hline $4 / 3$ & $10^{-4} r^{-3 / 2}$ & $\left(10^{-6} / 3\right) r^{-5 / 2}$ \\
\hline \multicolumn{5}{c}{ Grid extent } \\
radial, $r$ & azimuthal, $\theta$ & polar, $\phi$ & cells, $\left(N_{r}, N_{\theta}, N_{\phi}\right)$ \\
\hline$\left(0.8 r_{\mathrm{eh}}, 1,000 \mathrm{M}\right)$ & $(0.01 \pi, 0.99 \pi)$ & $(0,2 \pi)$ & $(256,128,128)$ \\
\hline \multicolumn{5}{c}{ Other parameters } \\
$a$ & $b$ & $l_{\text {torus, Kerr }}$ & $l_{\text {torus, dilaton }}$ \\
\hline 0.6 & 0.504 & 4.5 & 4.567 \\
\hline
\end{tabular}

Table 1: GRMHD simulation parameters, adapted from [9]. $r_{\mathrm{eh}}$ is the event horizon radius. The last row lists the Kerr dimensionless spin parameter, dilaton parameter and specific angular momenta. Note that $a$ and $b$ only exist in the Kerr and dilaton spacetimes, respectively.

Evolution (SANE) scenario [9, 14] in both black hole systems. Specifications of the GRMHD grid and fluid parameters are listed in Table 1.

The GRMHD simulations are carried out using the Black Hole Accretion Code BHAC [12] in modified Kerr-Schild coordinates. The GRMHD equations are evolved in a finite-volume representation. At each time step, we employ a piecewise-parabolic method, a total variation diminishing Lax-Friedrichs theme [11, 12] and a predictor-corrector scheme to update the fluid variables [12]. Further, the condition $\boldsymbol{\nabla} \cdot \boldsymbol{B}=0$ is handled by flux-interpolated constrained transport (FCT) [12]

\subsection{General-Relativistic Radiative Transfer (GRRT)}

We model synchrotron emission by carrying out GRRT calculations on the GRMHD simulations. Making use of the fast-light approximation, light rays (null geodesics) are integrated from a far-away observer to the black hole system. Subsequently, the intensity and optical depth equations are solved along each light ray [15]. Between $11000 \mathrm{M}$ and $12000 \mathrm{M}$ of the GRMHD simulations, 101 snapshots images are generated. The mass accretion rate is fixed at the $10000 \mathrm{M}$ snapshot to yield a flux of $3 \mathrm{Jy}$ at $230 \mathrm{GHz}$. Further, we choose $\mathrm{SgrA}^{*}$ as the model system with a mass of $M_{\mathrm{BH}}=4.148 \times 10^{6} \mathrm{M}_{\odot}$, at a distance of $D_{\mathrm{BH}}=8.175 \mathrm{kpc}$ [16].

The GRRT calculations are carried out with the Black Hole Observations in Stationary Spacetimes BHOSS code [17]. A Runge-Kutta-Fehlberg integrator handles the light rays, whereas the intensity and optical depth are simply updated in an Eulerian scheme [15]. The far-away observer is a plane perpendicular to the line of sight. For further details, see [18]. All GRRT calculation parameters are collected in table 2.

Since in the vicinity of black holes in active galactic nuclei various heating processes lead to different temperatures of electrons and protons, we relate the radiating electrons to the simulated protons by the "R- $\beta$-parametrization" ([19]; see also [20-22]):

$$
\frac{T_{\mathrm{p}}}{T_{\mathrm{e}}}=\frac{R_{\mathrm{low}}+R_{\mathrm{high}} \beta^{2}}{1+\beta^{2}} .
$$




\begin{tabular}{lllll}
\hline \hline & \multicolumn{3}{c}{ Imaging } \\
No. of pixels & width $/ r_{g}$ & width $/ \mu$ as & inclination & Target flux \\
\hline $1024 \times 1024$ & $40 \times 40$ & $211 \times 211$ & $60^{\circ}$ & 3 Jy \\
\hline \multicolumn{5}{c}{ Emission } \\
& $R_{\text {low }}$ & $R_{\text {high }}$ & eDF \\
\hline & 1 & $10,80,160$ & thermal \\
\hline
\end{tabular}

Table 2: GRRT calculation parameters. In the first row, $r_{g}=G M / c^{2}$.

In the above equation, $\beta \equiv p_{\text {gas }} / p_{\text {mag }}$ is the ratio of gas- to magnetic pressure. The parameters $R_{\text {high }}$ and $R_{\text {low }}$ control the temperature ratio in regions where $\beta \gg 1$ (the disk) and $\beta \ll 1$ (the jet), respectively. We set the synchrotron emission to be purely thermal. The corresponding electron distribution function reads [23]:

$$
\frac{d n_{\mathrm{e}}}{d \gamma_{\mathrm{e}} d \cos \xi d \phi}=\frac{n_{\mathrm{e}}}{4 \pi \Theta_{\mathrm{e}}} \frac{\gamma_{\mathrm{e}}\left(\gamma_{\mathrm{e}}^{2}-1\right)^{1 / 2}}{K_{2}\left(1 / \Theta_{\mathrm{e}}\right)} \exp \left(-\frac{\gamma_{\mathrm{e}}}{\Theta_{\mathrm{e}}}\right),
$$

with dimensionless electron temperature $\Theta_{\mathrm{e}}$, electron number density $n_{\mathrm{e}}$, gyrophase $\phi$, Lorentz factor $\gamma_{\mathrm{e}}$, pitch angle $\xi$ and modified Bessel function of the second kind $K_{2}$. Absorptivities and emissivities are taken from [24].

\section{Results}

The GRMHD simulations of the Kerr and dilaton black holes enter a relaxed state past $10000 \mathrm{M}$ and show very similar overall behavior. This was to be expected, since the two systems were matched at their ISCO. While the code accretion rates in the Kerr system are only slightly higher, the normalized magnetic flux through the horizon is doubled compared to the dilaton case. We define in the following the jet sheath as $0.1 \leq \sigma \leq 1.0 \wedge B e \geq 1.02$, where $B e=-h u_{t}$ is the Bernoulli parameter, $h$ is the specific enthalpy, and $u_{t}$ is the first component of the four-velocity. The magnetization is defined as $\sigma=B^{2} / \rho$, where $\rho$ is the local rest mass density and $B$ is the magnetic field stremgth. Lower magnetized regions we identify with the torus. Both black hole systems show an overall intermediate to low magnetized torus, where $\sigma \lesssim 10^{-2}$. In contrast to the dilaton black hole, the Kerr system shows a highly magnetized jet with a wider opening angle. This is due to more magnetic flux piling up near the horizon, caused by the Kerr black hole's rotation. As mentioned above, employing the R- $\beta$-parametrization leaves the (dimensionless) electron temperature $\Theta_{\mathrm{e}}$ in the jet unaltered, whereas an increase of $R_{\text {high }}$ leads to a decrease of electron temperature in the torus. For example, at $R_{\text {high }}=10$ electrons in the torus are hot $\left(\Theta_{\mathrm{e}} \sim 2.5\right)$, and much cooler for $R_{\text {high }}=160\left(\Theta_{\mathrm{e}} \sim 0.1\right)$.

Figure 1 shows averaged GRRT images for three values of $R_{\text {high }}$ in a square rooted scale, along with pixel-by-pixel differences between Kerr and dilaton black holes of the same emission model. The resulting total flux varies among models since we did not fix the total flux for the average image, but for a single snapshot. The R- $\beta$-parametrization essentially divides all GRRT images in torus-dominated $\left(R_{\text {high }}=10\right)$ and jet-dominated $\left(R_{\text {high }}>10\right)$ models. The torus in the former class of models extends further inward and outward for the dilaton black hole. On the one hand, 

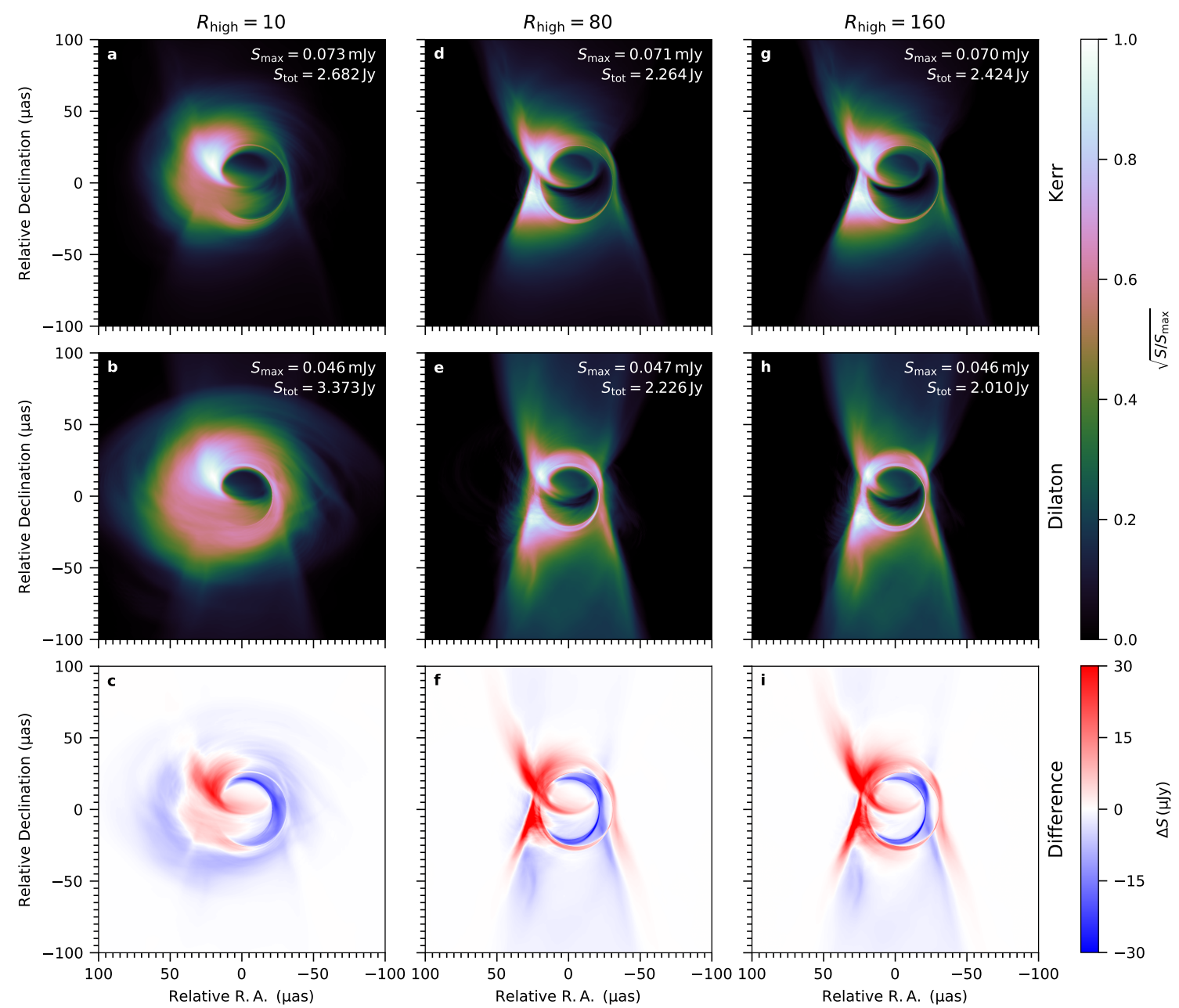

Figure 1: Effect of the R- $\beta$-parametrization (Eq. 4) on averaged GRRT images of a Kerr black hole (top row) and a dilaton black hole (middle row), along with pixel-by-pixel differences (bottom row). The top and middle rows are shown in a square rooted color scale to highlight low flux features. Images are generated with thermal synchrotron radiation at $230 \mathrm{GHz}$ and averaged over 101 snapshots taken between $11,000 \mathrm{M}$ to $12,000 \mathrm{M}$ of the GRMHD simulation.

there is more distance to cover for accreted matter. This is due to the smaller event horizon of the dilaton black hole caused by the ISCO matching. On the other hand, increased Doppler beaming around the Kerr black hole leads to significant differences in the brightness distributions between the spacetimes in the torus-dominated configurations (see panels (a) and (e) of Fig. 1).

In the Kerr image (panel a), the approaching limb is very prominent, while the receding limb is barely visible. The region around the point of peak flux north-west of the shadow shows a sharp boundary past $60 \%$ of the peak. The adjacent lower-flux region merges into an arc of emission from the torus and the jet foot-point spanning halfway across the Kerr shadow. South-west of the shadow, the faint onset of the counter-jet is visible. In the dilaton spacetime, the receding limb is much more prominent, and the aforementioned $60 \%$ flux region is not as clearly cut as in the Kerr system. 
The pixel-by-pixel differences again highlight prominent Doppler boosted emission on the approaching side of the Kerr system especially close to the shadow, while the dilaton system exhibits filamentary structures from $\sim 30 \mu$ as from the center outwards.

For $R_{\text {high }}=80$, the source structure changes fundamentally from a torus-dominated system towards a jet onset extending outwards into filaments (middle column of Fig. 1), showing the wider opening angle in the Kerr system. For both spacetimes, the most of the emission comes from two "hot-spots" located at the northern and southern halves of the approaching side close to the shadow. Overall, the source structures of Kerr- and dilaton images are much more similar than for low $R_{\text {high }}$. The arc of emission discussed above reduces to the part that traces the foot of the observer-facing jet. Pixel-by-pixel differences (panels $f$ and i) further illustrate the filamentary structures and show how the effects of Doppler boosting affect the region close to the shadow, its apparent size and the jet onset.

Increasing $R_{\text {high }}$ to 160 , the source structure remains unaltered (rightmost column of Fig. 1); the electron temperature in the torus at $R_{\text {high }}=80$ is already too low for an additional decrease to make a noticeable difference in the images.

\section{Summary and Conclusion}

In this work, we carried out GRRT calculations on GRMHD simulations of a Kerr- and a dilaton black hole, employing a parametrization for the electron temperature. For each emission model, we compare the images between the two spacetimes. In the GRRT images, the black hole shadows are clearly distinguishable, since the event horizons of the two black holes are of different sizes due to the ISCO match. The Kerr shadow is asymmetric and off-centered, while the the dilaton shadow is circular and smaller. The $\mathrm{R}-\beta$-parametrization splits the source morphologies into torus- and jet dominated images, where the former show the largest differences between spacetimes. Mainly, the torus is more prominent in the dilaton system due to the reduced Doppler boosting compared to the Kerr case. The jet on the other hand shows a larger opening angle and higher magnetization in the Kerr spacetime.

Clearly, GRRT calculations lead to "infinite"-resolution images not reflecting a real-world observing scenario; interpretations of these images are therefore to be drawn with care. Images obtained from observations are limited by the telescope resolution and other image-corrupting effects, and are therefore blurred compared to the GRRT images we derive from our simulations. In order to assess whether Kerr- and dilaton black holes can be distinguished in an actual observation, synthetic VLBI data has to be generated from the GRRT images, mimicking an observation campaign. We can thereby test how future observations with instruments such as the Event Horizon Telescope can help probing gravity in the strong-field regime. Further, we expect electrons in the jet to be accelerated by magnetic reconnection, requiring a non-thermal electron energy distribution function during the GRRT calculations. Both the constraints of an observation and aforementioned emission models will be subject to investigation in a forthcoming publication. 


\section{Acknowledgments}

This research is supported by the ERC synergy grant "BlackHoleCam: Imaging the Event Horizon of Black Holes" (Grant No. 610058). Christian M. Fromm is supported by the Black Hole Initiative at Harvard University, which is supported by a grant from the John Templeton Foundation. Ziri Younsi is supported by a UKRI Stephen Hawking Fellowship and acknowledges support from a Leverhulme Trust Early Career Fellowship. The simulations were performed on GOETHE-HLR LOEWE at the CSC-Frankfurt and Iboga at ITP Frankfurt.

\section{References}

[1] G.A. Shields, A Brief History of Active Galactic Nuclei, PASP 111 (1999) 661 [astro-ph/9903401].

[2] A. Eckart and R. Genzel, Observations of stellar proper motions near the Galactic Centre, Nature 383 (1996) 415.

[3] A.M. Ghez, B.L. Klein, M. Morris and E.E. Becklin, High Proper-Motion Stars in the Vicinity of Sagittarius A*: Evidence for a Supermassive Black Hole at the Center of Our Galaxy, Astrophys. J. 509 (1998) 678 [astro-ph/9807210].

[4] The LIGO Scientific Collaboration and the Virgo Collaboration, Observation of Gravitational Waves from a Binary Black Hole Merger, Phys. Rev. Lett. 116 (2016) 061102 [1602.03837].

[5] B. Boccardi, T.P. Krichbaum, E. Ros and J.A. Zensus, Radio observations of active galactic nuclei with mm-VLBI, Astronomy and Astrophysics Reviews 25 (2017) 4 [1711.07548].

[6] Event Horizon Telescope Collaboration, K. Akiyama, A. Alberdi, W. Alef, K. Asada, R. Azulay et al., First M87 Event Horizon Telescope Results. I. The Shadow of the Supermassive Black Hole, Astrophys. J. Lett. 875 (2019) L1.

[7] A. Einstein, Naherungsweise Integration der Feldgleichungen der Gravitation, Sitzungsberichte der Königlich Preussischen Akademie der Wissenschaften (Berlin) 1916 (1916) 688.

[8] E. Asmodelle, Tests of General Relativity: A Review, arXiv e-prints (2017) arXiv:1705.04397 [1705.04397].

[9] Y. Mizuno, Z. Younsi, C.M. Fromm, O. Porth, M. De Laurentis, H. Olivares et al., The current ability to test theories of gravity with black hole shadows, Nature Astronomy 2 (2018) 585 [1804.05812].

[10] A. García, D. Galtsov and O. Kechkin, Class of Stationary Axisymmetric Solutions of the Einstein-Maxwell-Dilaton-Axion Field Equations, Phys. Rev. Lett. 74 (1995) 1276.

[11] L. Rezzolla and O. Zanotti, Relativistic Hydrodynamics, Oxford University Press, Oxford, UK (2013), 10.1093/acprof:oso/9780198528906.001.0001. 
[12] O. Porth, H. Olivares, Y. Mizuno, Z. Younsi, L. Rezzolla, M. Moscibrodzka et al., The black hole accretion code, Computational Astrophysics and Cosmology 4 (2017) 1 [1611.09720].

[13] J.A. Font and F. Daigne, On the stability of thick accretion disks around black holes, Astrophys. J 581 (2002) L23.

[14] R. Narayan, A. Sagdowski, R.F. Penna and A.K. Kulkarni, GRMHD simulations of magnetized advection-dominated accretion on a non-spinning black hole: role of outflows, Mon. Not. R. Astron. Soc. 426 (2012) 3241 [1206. 1213].

[15] Z. Younsi, K. Wu and S.V. Fuerst, General relativistic radiative transfer: formulation and emission from structured tori around black holes, Astron. Astrophys. 545 (2012) A13 [1207.4234].

[16] Gravity Collaboration, R. Abuter, A. Amorim, M. Bauböck, J.P. Berger, H. Bonnet et al., A geometric distance measurement to the Galactic center black hole with $0.3 \%$ uncertainty, Astron. Astrophys. 625 (2019) L10 [1904.05721].

[17] Z. Younsi, O. Porth, Y. Mizuno, C.M. Fromm and H. Olivares, Modelling the polarised emission from black holes on event horizon-scales, in Perseus in Sicily: From Black Hole to Cluster Outskirts, K. Asada, E. de Gouveia Dal Pino, M. Giroletti, H. Nagai and R. Nemmen, eds., vol. 342, pp. 9-12, Jan., 2020, DOI [1907.09196].

[18] Z. Younsi, A. Zhidenko, L. Rezzolla, R. Konoplya and Y. Mizuno, New method for shadow calculations: Application to parametrized axisymmetric black holes, Phys. Rev. D 94 (2016) 084025 [1607 . 05767].

[19] M. Mościbrodzka, H. Falcke and H. Shiokawa, General relativistic magnetohydrodynamical simulations of the jet in M 87, Astron. Astrophys. 586 (2016) A38 [1510. 07243].

[20] A. Cruz-Osorio, C.M. Fromm, Y. Mizuno, A. Nathanail, Z. Younsi, O. Porth et al., State-of-the-art energetic and morphological modelling of the launching site of the M87 jet, Nature Astronomy (2021) [2111.02517].

[21] C.M. Fromm, A. Cruz-Osorio, Y. Mizuno, A. Nathanail, Z. Younsi, O. Porth et al., Impact of non-thermal particles on the spectral and structural properties of M87, arXiv e-prints (2021) arXiv:2111.02518 [2111.02518].

[22] J. Davelaar, H. Olivares, O. Porth, T. Bronzwaer, M. Janssen, F. Roelofs et al., Modeling non-thermal emission from the jet-launching region of $M 87$ with adaptive mesh refinement, Astron. Astrophys. 632 (2019) A2 [1906. 10065].

[23] P.K. Leung, C.F. Gammie and S.C. Noble, Numerical Calculation of Magnetobremsstrahlung Emission and Absorption Coefficients, Astrophys. J. 737 (2011) 21.

[24] A. Pandya, Z. Zhang, M. Chandra and C.F. Gammie, Polarized Synchrotron Emissivities and Absorptivities for Relativistic Thermal, Power-law, and Kappa Distribution Functions, Astrophys. J. 822 (2016) 34 [1602 . 08749]. 